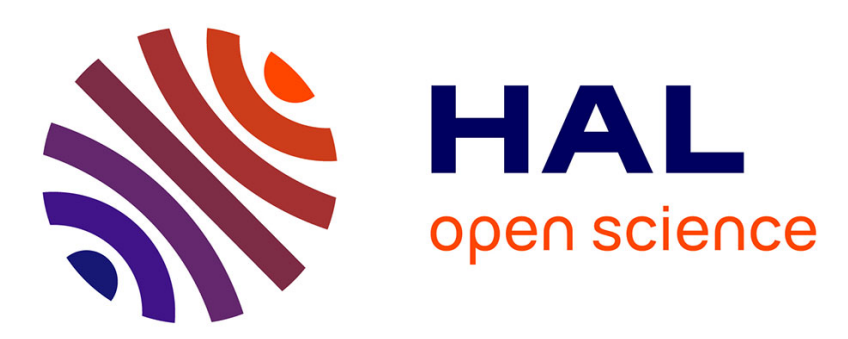

\title{
Discharge impedance evolution, stray capacitance effect, and correlation with the particles size in a dusty plasma
}

Gaëtan Wattieaux, Laïfa Boufendi

\section{To cite this version:}

Gaëtan Wattieaux, Laïfa Boufendi. Discharge impedance evolution, stray capacitance effect, and correlation with the particles size in a dusty plasma. Physics of Plasmas, 2012, 19 (3), pp.033701. 10.1063/1.3689013 . insu-01258778

\section{HAL Id: insu-01258778 \\ https://hal-insu.archives-ouvertes.fr/insu-01258778}

Submitted on 19 Jan 2016

HAL is a multi-disciplinary open access archive for the deposit and dissemination of scientific research documents, whether they are published or not. The documents may come from teaching and research institutions in France or abroad, or from public or private research centers.
L'archive ouverte pluridisciplinaire HAL, est destinée au dépôt et à la diffusion de documents scientifiques de niveau recherche, publiés ou non, émanant des établissements d'enseignement et de recherche français ou étrangers, des laboratoires publics ou privés. 


\section{AIP | Physics of

Discharge impedance evolution, stray capacitance effect, and correlation with the particles size in a dusty plasma

Gaëtan Wattieaux and Laïfa Boufendi

Citation: Physics of Plasmas 19, 033701 (2012); doi: 10.1063/1.3689013

View online: http://dx.doi.org/10.1063/1.3689013

View Table of Contents: http://scitation.aip.org/content/aip/journal/pop/19/3?ver=pdfcov

Published by the AIP Publishing

Articles you may be interested in

Observation of $\Omega$ mode electron heating in dusty argon radio frequency discharges

Phys. Plasmas 20, 083704 (2013); 10.1063/1.4818442

Optical and kinetic properties of the dusty plasma in radiofrequency discharge

Phys. Plasmas 19, 023706 (2012); 10.1063/1.3690103

Measurements of time average series resonance effect in capacitively coupled radio frequency discharge plasma

Phys. Plasmas 18, 103509 (2011); 10.1063/1.3646317

The response of a capacitively coupled discharge to the formation of dust particles: Experiments and modeling Phys. Plasmas 13, 073507 (2006); 10.1063/1.2222258

Detection of particles of less than $5 \mathrm{~nm}$ in diameter formed in an argon-silane capacitively coupled radiofrequency discharge

Appl. Phys. Lett. 79, 4301 (2001); 10.1063/1.1425431

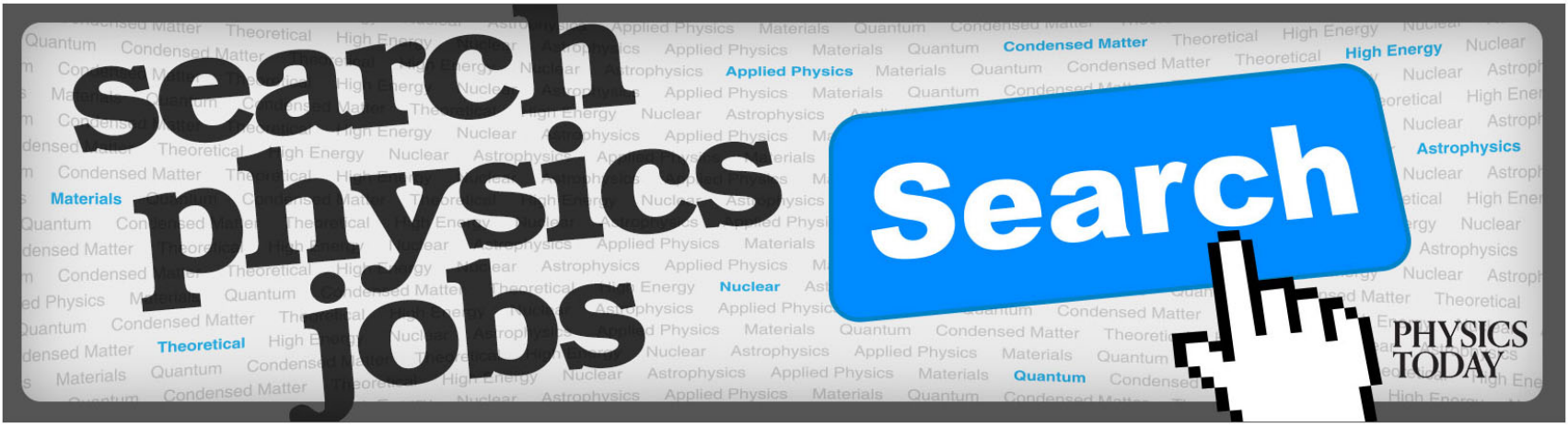




\title{
Discharge impedance evolution, stray capacitance effect, and correlation with the particles size in a dusty plasma
}

\author{
Gaëtan Wattieaux ${ }^{1}$ and Laïfa Boufendi ${ }^{2}$ \\ ${ }^{1}$ LPC2E, Orleans University, $3 A$ Avenue de la Recherche Scientifique, 45071 Orléans cedex 2, France \\ ${ }^{2}$ GREMI, Orleans University, 14 rue d'Issoudun BP 6744, 45067 Orléans cedex 2, France
}

(Received 13 December 2011; accepted 31 January 2012; published online 5 March 2012)

Dust particles growing or injected in a plasma modify significantly the impedance of capacitively coupled radio frequency discharges. The principal modifications are the increase of the plasma bulk resistance and of the plasma sheath capacitance. In this work, we propose a method to evaluate the impedance of the discharge (sheath + plasma bulk) during the growth of dust particles in a plasma. This method does not require the measurement of any current/voltage phase shift. Then, the evolution of the power coupled into the plasma as well as the voltage drop across the plasma bulk are derived. It follows that the plasma coupled power increases by a factor of five during the dust growth. The effect of the reactor stray capacitance on the power coupled to the plasma is underlined. Finally, a perfect correlation between the evolution of the size of the dust particles in the plasma and the increase of the plasma/electrode sheath capacitance suggests that charged dust particles induce an electrostatic force on the plasma sheath. An analytical model is proposed in order to take this phenomenon into account in future dusty plasma electrical modelling.

(C) 2012 American Institute of Physics. [http://dx.doi.org/10.1063/1.3689013]

\section{INTRODUCTION}

Plasma induced dust production in capacitively coupled radio frequency (CCRF) discharges has been and is still being widely studied and the span of the researches is very broad. ${ }^{1-6}$ Sometimes, the occurrence of the dusts in the discharge is studied in order to avoid it such as it is the case for microelectronics applications ${ }^{7,8}$ where dusts are one of the most important source of irremediable defects on microchips. ${ }^{9,10}$ On the contrary, the production of dust can be desired in order to create new materials with outstanding performances such as thin film solar cells, ${ }^{11}$ super hard materials, ${ }^{12}$ self lubricating coatings, ${ }^{13}$ or nanocrystalline diamonds. ${ }^{14}$ Besides, the ability to measure the size and the concentration of dust particles using a CCRF discharge has been underlined recently. ${ }^{15,16}$

All these applications have at least one common characteristic: The electrical properties of the discharge are affected by the presence of the dust particles in the plasma. This effect has been successfully used to detect the occurrence of dust formation in a discharge and to follow the different dust growth phases measuring the self-bias voltage of the powered electrode or the discharge current. ${ }^{17}$ Unfortunately, this empirical diagnostic has to be adapted from one reactor to an other, for example, changing the monitored current harmonics.

The initial aim of this work is to go deeper into the study of the influence of dusts on the electrical properties of the discharge such as the plasma coupled power or the voltage across the plasma bulk. To meet this requirement, we particularly focused on the discharge impedance evolution. At the operating frequency $(13.56 \mathrm{MHz})$, most of this impedance is due to the plasma sheath but the plasma bulk impedance influence is not negligible especially when dust particles are produced in the plasma. Moreover, the electrode sheath thickness evolution changes during the dust formation. This phenomenon, which is observable with the naked eye in the presence of an important dust concentration in the plasma, means that the electrode sheath capacitance is modified by the presence of dust particles in the discharge.

Measuring accurately, the electrical parameters of capacitively coupled radio frequency discharges (i.e., the plasma coupled power or the discharge current) have always been a crucial point in this research area and simple methods are required for experimental applications. Usually, subtractive method is used to determine the power coupled to the plasma. The purpose of this approach is to evaluate the plasma coupled power by subtracting the power consumed in the impedance matching circuit from the power provided by the generator. ${ }^{18}$ Such a method has been recently proposed by Nelis et al. ${ }^{19}$ following Canpont ${ }^{20}$ and Marshall et al. ${ }^{21}$ Using a different approach, the originality of our method is to study the impedance of the discharge without measuring the current voltage phase shift at the electrode connected to the radiofrequency source (active electrode). The obtained results are compared with an other work carried out under similar conditions. Moreover, this approach helped us to point out the effect of the stray capacitance on the power coupled to the plasma during the dust growth and we noticed that the evolution of the electrode sheath capacitance is strongly correlated with the evolution of the dust particle size.

This paper is organized as follows: Sec. II is dedicated to the presentation of the experimental setup; in Sec. III, we present how we calculate the plasma bulk impedance during the dust growth from the measurement of the mean electron density in the discharge; in Sec. IV, the circuit calibration protocol is presented; Sec. V concerns the evolution of the electrical parameters of the discharge during the dust growth; and Sec. VI is a discussion about the evolution of the electrode sheath capacitance compared with other electrical parameters and with the evolution of the size of the particles in the discharge. Finally, we will conclude this contribution. 


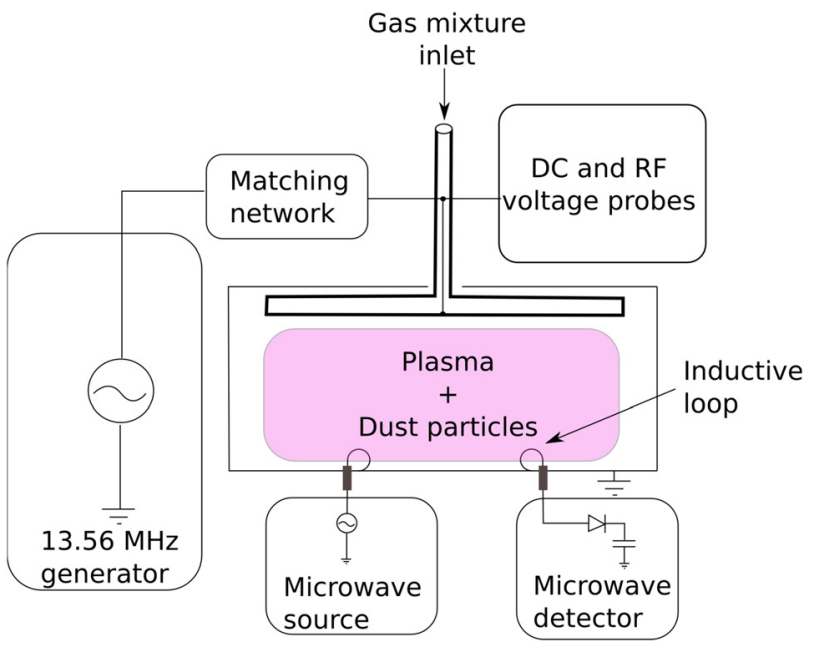

FIG. 1. (Color online) Schematic of the experiment

\section{EXPERIMENTAL SETUP}

The CCRF discharge is carried out into a cylindrical discharge box $(13 \mathrm{~cm}$ diameter, $3.3 \mathrm{~cm}$ height $)$ operating at low pressure $(100 \mathrm{~Pa})$ which has been widely described elsewhere. ${ }^{22}$ Dusts are produced in the plasma phase from a $98 \%$ $\mathrm{Ar} / 2 \%$ methane initial gas mixture. This reactor is coupled to a $13.56 \mathrm{MHz}$ generator through a L-type matching network (see Figs. 1 and 2). The series (blocking) capacitance $C_{3}$ at the output of this circuit makes it possible to induce a DC self-bias at the active electrode when the discharge is ignited. The electrical potential of this electrode is measured using a voltage probe for the alternating AC part and a low-pass filter for its DC value. The electron density is measured from the plasma induced shift of the TM010 $(1.7 \mathrm{GHz})$ resonance mode of the discharge box. ${ }^{23,24}$ The stray capacitance $C_{2}$ of the active electrode is $538 \mathrm{pF}$ and has been measured with a capacitance meter. The discharge is switched on during $20 \mathrm{~s}$. After this duration, the mean diameter of the spherical dust particle formed in the discharge reaches $200 \mathrm{~nm}$ with $48 \mathrm{~nm}$ standard deviation. ${ }^{15}$ In the mean time, the electron density, the amplitude of the radio frequency voltage applied on the active electrode (top electrode), and the self-bias voltage are particularly affected (see Figs. 3 and 4). Moreover, the $750.4 \mathrm{~nm}$ argon line is recorded. As we can see in Figure 5, the intensity of this atomic line increases by a factor of 4 to 5 during the dust growth. This radiative transition has a probability of $4.45 \times 10^{7} \mathrm{~s}^{-1}$ (Ref. 25) and corresponds to the spontaneous transition of the argon $2 p_{1}\left(3 s^{2} 3 p^{5}\left({ }^{2} P_{1 / 2}^{0}\right)\right)$ exited level $(13.48 \mathrm{eV})$ to the $1 s_{2}\left(3 s^{2} 3 p^{5}\left({ }^{2} P_{3 / 2}^{0}\right)\right)$ state $(11.83 \mathrm{eV})$. An other $2 p_{1} \rightarrow 1 s_{4}$ radiative transition occurs at $667.7 \mathrm{~nm}$ with an associated probability of $2.36 \times 10^{5} \mathrm{~s}^{-1}$ (Ref. 25). Consequently, the $750.4 \mathrm{~nm}$ argon atomic line intensity is 200 times greater than the $667.7 \mathrm{~nm}$ argon atomic line which will be neglected in this study. Moreover, we assume that the plasma electron density is negligible compared to the neutrals (which are considered as argon atoms), the electron temperature is rather weak and the pressure in the reactor is low enough to neglect any quenching effects for argon exited atoms. Thus, we can consider that most of the argon neutrals are in fundamental state and the $2 p_{1}$ level is mostly depopulated through the $2 p_{1} \rightarrow 1 s_{2}$ spontaneous transition. ${ }^{26}$ Finally, the auto-absorption is neglected and the electrons/neutrals collisions remain the only argon neutrals excitation source. This situation is characteristic of a coronal equilibrium and is described at steady state by the following relationship:

$$
n_{e} n_{A r} k_{e x c}=\frac{n_{2 p_{1}}}{\tau},
$$

with $n_{e}$ is the electron density, $n_{A r}=2.3 \times 10^{22} \mathrm{~m}^{-3}$ is the argon neutrals density which is determined from the pressure in the reactor, $k_{e x c}$ is the neutral argon excitation coefficient corresponding to a transition from fundamental to level $2 p_{1}$ by electron/neutral collisions, $\tau \approx 22 \mathrm{~ns}$ is the life time of the $2 p_{1}$ excited state, and $n_{2 p 1}$ is the population density of this level. This density is proportional to the intensity of the $750.4 \mathrm{~nm}$ argon line and it has been determined after having performed the energy calibration of the spectrometer thanks to a tungsten ribbon filament lamp. We find this density to be $1.21 \times 10^{11} \mathrm{~m}^{-3}$ at the ignition of the discharge and it reaches $4 \times 10^{11} \mathrm{~m}^{-3}$ after 20 s (see Fig. 5). The relationship between the excitation coefficient and the electron temperature has been calculated by Gordillo-Vasquez et al..$^{27}$

$$
k_{\text {exc }} \approx 2.78 \times 10^{-15} \exp \left(-13.5 / T_{e}(V)\right) .
$$

Combining Eqs. (1) and (2), we obtain the following expression for the electron temperature:

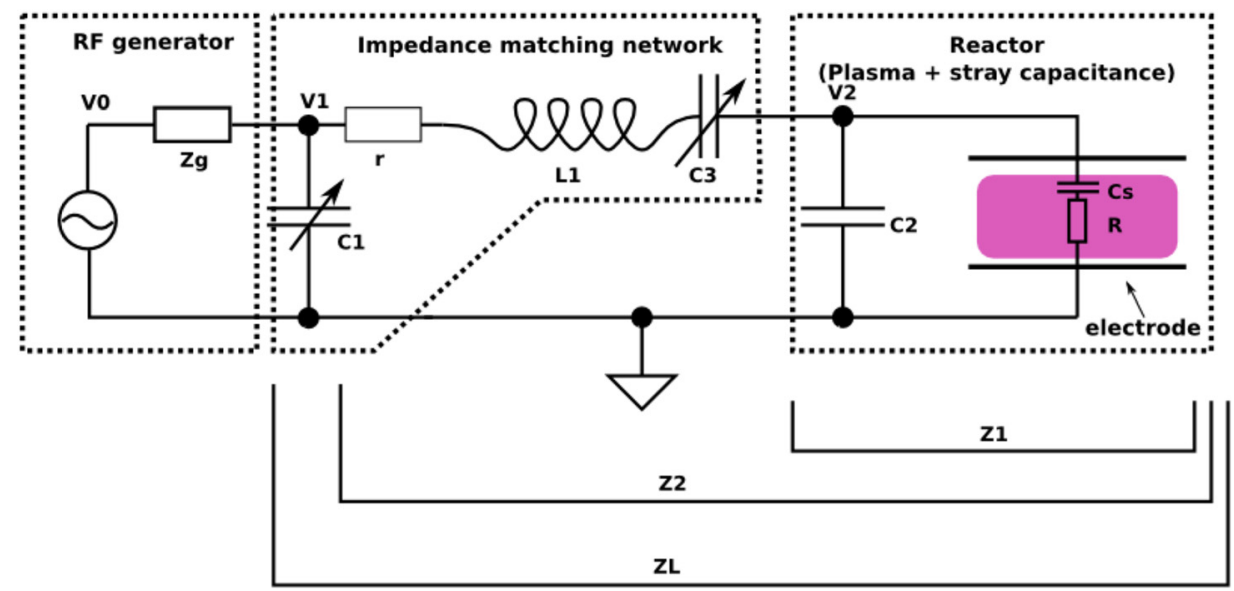

FIG. 2. (Color online) Electrical equivalent circuit of the experiment. 


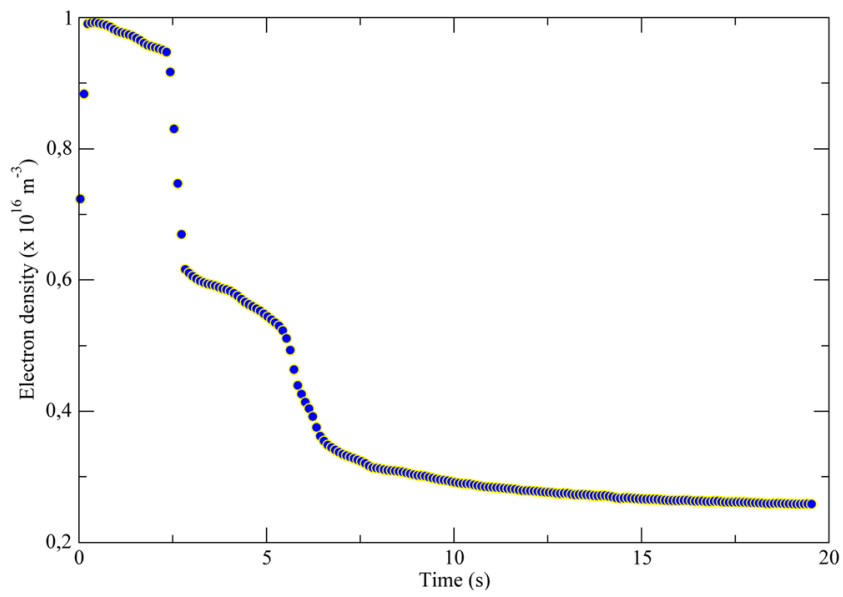

FIG. 3. (Color online) Time evolution of the electron density.

$$
T_{e}(V) \approx-\frac{13.5}{\ln \left(0.7 \frac{n_{2 p_{1}}}{n_{e}}\right)}
$$

Using this equation, the electron temperature evolution can be determined during the dust growth. This result is presented in Figure 6 showing a 30\% increase of the electron temperature during the process.

\section{IMPEDANCE OF THE PLASMA BULK}

In order to simplify the study, it is useful to model the discharge (sheath + plasma bulk) as a series connection of a resistor and a capacitor and to consider only the fundamental frequency $(13.56 \mathrm{MHz})$ of the applied voltage at the active electrode. $^{28}$ The resistor represents the plasma bulk whereas the capacitance represents the electrode/plasma sheath. This hypothesis is justified if the angular frequency $\omega$ of the voltage at the active electrode is small enough compared to the electron/neutral collision frequency $\nu$ which is our case: $\omega=8.52 \times 10^{7} \mathrm{rad} \cdot \mathrm{s}^{-1}$ and $\nu \approx 8 \times 10^{8} \mathrm{rad} \cdot \mathrm{s}^{-1}$ in argon at $100 \mathrm{~Pa}$ (Ref. 18). According to this simple model, the discharge impedance can be approximated by

$$
Z_{p}=R+\frac{1}{j C_{s} \omega},
$$

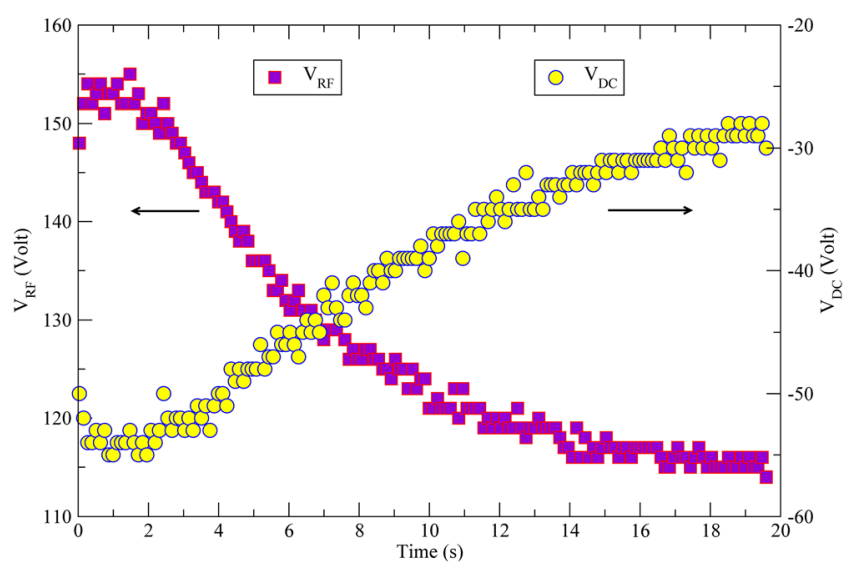

FIG. 4. (Color online) Active electrode voltage amplitude $\left(V_{R F}\right)$ and selfbias voltage $\left(V_{D C}\right)$ temporal evolution.

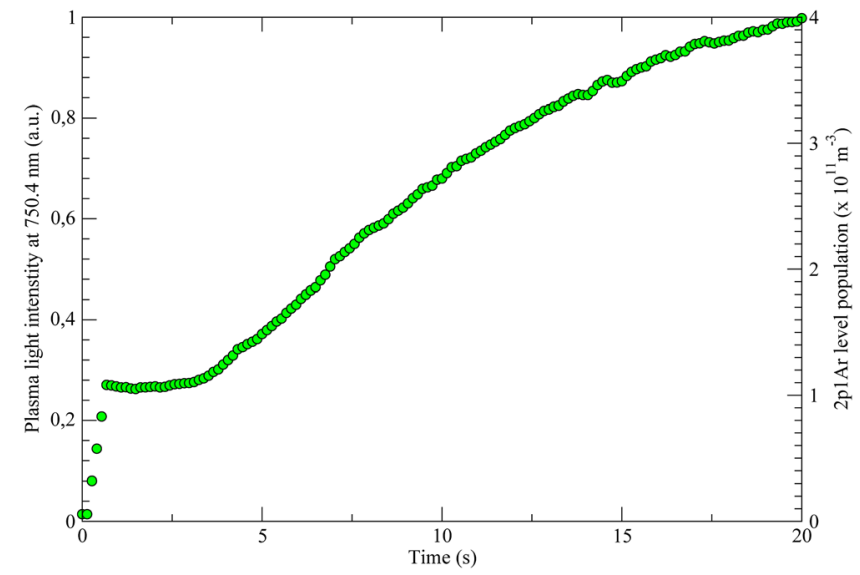

FIG. 5. (Color online) Plasma emission at $750.4 \mathrm{~nm}$ and population of the state $2 p_{1}$ versus time.

with $C_{s}$ the electrode/plasma sheath and $R$ being the real part of the plasma bulk impedance

$$
\begin{aligned}
& R=\mathbb{R}\left(\frac{1}{j \omega \varepsilon_{0}} \int_{0}^{h} \frac{d x}{\int_{0}^{S} \varepsilon_{r}(x, a) d S}\right), \\
& =\mathbb{R}\left(\frac{1}{2 j \omega \varepsilon_{0} \pi} \int_{0}^{h} \frac{d x}{\int_{0}^{h / 2} a \cdot \varepsilon_{r}(x, a) d a}\right),
\end{aligned}
$$

where $x$ and $a$ correspond, respectively, to the axis and to the radius vectors of the cylindrical discharge box, $\varepsilon_{0}$ and $\varepsilon_{r}$ are, respectively, the permittivity in vacuum and the relative permittivity of the plasma, $h=3.3 \mathrm{~cm}$ and $d=13 \mathrm{~cm}$ are, respectively, the height and the diameter of the discharge box. The relative permittivity of the plasma bulk is given by $^{23,24}$

$$
\varepsilon_{r}(x, a) \approx 1-3.18 \times 10^{4} \cdot n_{e}(x, a) \frac{1+j \frac{\nu}{\omega}}{\nu^{2}+\omega^{2}},
$$

with $n_{e}(x, a)$ the electron density that is supposed to present a collisional regime (cosine) spatial profile ${ }^{18}$

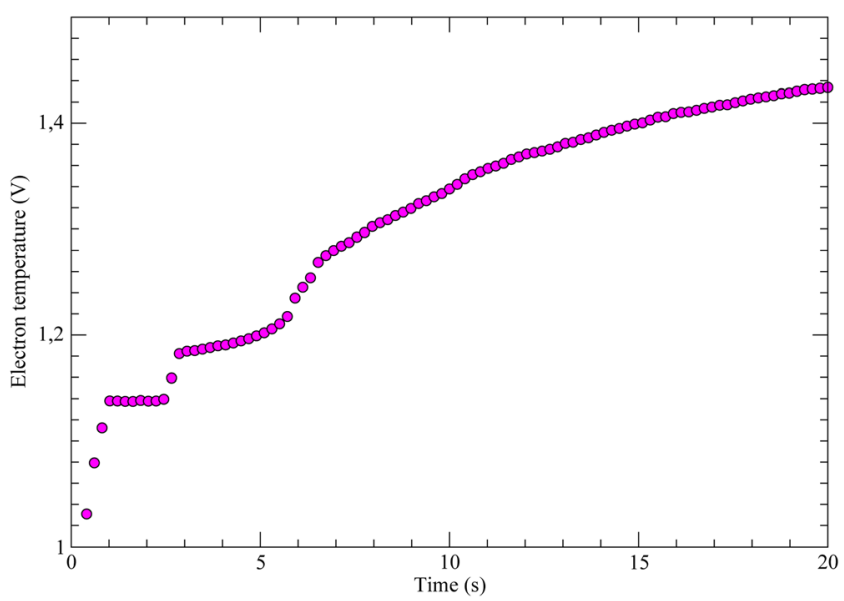

FIG. 6. (Color online) Evolution of the electron temperature versus time. 


$$
n_{e}(x, a)=2.5 \times \overline{n_{e}} \cdot \sin \left(\frac{\pi x}{h}\right) \cos \left(\frac{\pi a}{d}\right)
$$

where $\overline{n_{e}}$ corresponds to the mean electron density determined by microwave resonant cavity measurement (see Fig. 3).

The strong increase of the resistive part of the discharge during the dust growth is presented in Figure 7. If we look at the ignition of the discharge, the plasma bulk resistance is found to be around $25 \mathrm{Ohms}$. This value is in a good agreement with the work of Schauer et al. under similar conditions. ${ }^{29}$

As it will be presented in Sec. IV, this method for the determination of the plasma bulk resistance offers the possibility to analyze the evolution of the discharge impedance without measuring neither the discharge current nor the current/voltage phase shift which is particularly convenient because this last measurement is rather difficult to be performed accurately at $13.56 \mathrm{MHz}$.

\section{CALIBRATION WITHOUT DUST}

In order to perform our analysis, we need to determine every element of the load circuit. More precisely, we want to know the value of $r$, the series resistance of the matching circuit, and of $L=L_{1}-\left(C_{3} \omega^{2}\right)^{-1}$, the series inductance of this circuit. This calibration can be performed in two steps, $r$ and $L$ being respectively determined without and with plasma in the reactor.

The impedance of the load circuit is given by (see Fig. 2)

$$
Z_{L}=\left(j C_{1} \omega+Z_{2}^{-1}\right)^{-1}
$$

with

$$
Z_{2}=r+j L \omega+Z_{1}
$$

and

$$
Z_{1}=\left(j C_{2} \omega+\left(R+\left(j C_{s} \omega\right)^{-1}\right)^{-1}\right)^{-1}
$$

with $C_{2}=538 p F$ the stray capacitance of the reactor, $C_{1}$ the load capacitance of the matching network, $R$ the plasma bulk resistance, and $C_{S}$ the plasma sheath capacitance.

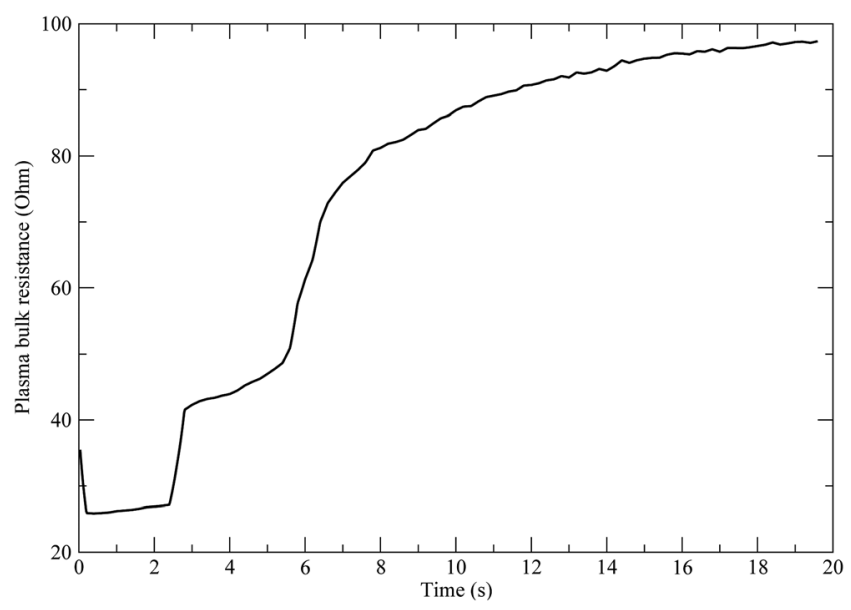

FIG. 7. Evolution of the plasma bulk resistance during the dust growth.
There are two resonances for $Z_{L}: \omega_{1}$ for which $Z_{L}$ is minimum and $\omega_{2}$ for which $Z_{L}$ is maximum. In order to induce a dielectric breakdown between the electrodes of the reactor, we need to apply a high voltage on the active electrode. The best condition is obtained when the frequency delivered by the RF generator matches with $\omega_{2}$ and when the impedance matching between the generator and the load is achieved. To meet this requirement, one have to adjust $C_{1}$ and $C_{3}$.

\section{A. Determination of $r$}

The series resistance of the circuit is determined adjusting the pressure in the reactor in order to avoid the ignition of the plasma between the electrodes. The impedance matching is then achieved adjusting $L$ and $C_{1}$ for a given power of $30 \mathrm{~W}$. Then, $C_{1}=1.64 \mathrm{nF}$ is determined using a capacitance meter. This capacitance represents the capacitance of the parallel capacitor of the matching circuit and the capacitance of the cable starting from the generator to the matching box. Moreover, we check that the highest voltage applied on the active electrode is obtained when the impedance matching is achieved meaning that $\omega_{2}=\omega=85.2 \times 10^{6} \mathrm{rad} \cdot \mathrm{s}^{-1}$ when $Z_{L}=Z_{g}=50 \Omega, \omega$ and $Z_{g}$ being, respectively, the excitation angular frequency and the output impedance of the generator. In these conditions, we have

$$
\omega_{2}=\sqrt{\frac{C_{1}+C_{2}}{L C_{1} C_{2}}},
$$

and the impedance of the load circuit reduces to

$$
Z_{L}=\frac{1}{\left(C_{1} \omega\right)^{2}}\left(\frac{1}{r}-j C_{1} \omega\right) \approx 50 \Omega,
$$

under usual conditions, $\frac{1}{r} \gg C_{1} \omega$, thus we find

$$
r \approx \frac{1}{50\left(C_{1} \omega\right)^{2}} \approx 1.02 \Omega .
$$

\section{B. Determination of $L$}

In a second time, a pure argon plasma is ignited in the chamber at a pressure of $100 \mathrm{~Pa}$. As a consequence, the impedance of the load circuit has to be readjusted due to the influence of the plasma impedance. This task is achieved by adjusting $L$ (through $C_{3}$ ) whereas $C_{1}$ is kept constant. Then, $\widehat{V_{2}}$, the $\mathrm{AC}$ voltage amplitude on the active electrode reaches $153 \mathrm{~V}$. Using Eqs. (9)-(11), it is straightforward to get the expression of $\widehat{V_{2}}$ when the impedance matching is achieved $\left(Z_{L}=Z_{g}\right)$

$$
\widehat{V_{2}}=\widehat{V_{0}}\left|\frac{Z_{1} Z_{L}}{\left(Z_{L}+Z_{g}\right) Z_{2}}\right| \text {, }
$$

where $\widehat{V_{0}}=\sqrt{8 P_{g} Z_{g}} \approx 110 \mathrm{~V}$ is twice the output voltage amplitude of the generator connected to a tuned load $\left(P_{g}\right.$ is the output power delivered by the generator).

At first glance, it could appear difficult to get the value of $L$ from Eq. (15) because $C_{s}$, the plasma sheath capacitance, is also unknown (plasma bulk resistance has been determined in Sec. III). Remembering that the tuned load has a 50 Ohms 
impedance, we get a second equation and the problem can be solved. As shown in Figure 8, there is only one couple $\left(C_{s} \approx 28 \mathrm{pF}, L \approx 329 \mathrm{nH}\right)$ in our experimental conditions which is compatible with a $153 \mathrm{~V}$ RF voltage amplitude at the active electrode.

We can now compute the power coupled to the load

$$
P_{l}=\left(\frac{\widehat{V_{2}}}{\sqrt{2}}\right)^{2}\left|\frac{Z_{2}}{Z_{1} Z_{L}}\right| \cos \left(\varphi_{L}\right) \approx 29.9 \mathrm{~W},
$$

with $\left|Z_{L}\right|$ and $\varphi_{L}$ being, respectively, the modulus and the argument of $Z_{L}$, the load impedance.

Finally, the power which is effectively coupled into the plasma is given by

$$
P_{p}=\frac{\left(\widehat{V_{2}}\right)^{2}}{2\left|Z_{p}\right|} \cos \left(\varphi_{p}\right) \approx 2 \mathrm{~W},
$$

with $\left|Z_{p}\right|=\sqrt{R^{2}+\left(C_{s} \omega\right)^{-2}}$ and $\varphi_{p}=-\arctan \left(\left(R C_{s} \omega\right)^{-1}\right)$ being, respectively, the modulus and the argument of $Z_{p}$, the discharge impedance (sheath + plasma). We notice that only $7 \%$ of the power delivered by the generator ends up in the plasma, the rest is dissipated in the matching network, essentially through $r$.

The electrical parameters of the circuit which have been determined for a dust-free plasma are summarized in Table I. At this point, every element of the electrical circuit has been determined for a dust-free discharge. Let us see now what happen when dust particles appear in the plasma.

\section{ELECTRICAL PARAMETERS EVOLUTION DURING THE DUST GROWTH}

After the impedance matching is accurately adjusted for a pure argon discharge, methane is injected in the chamber and the discharge impedance begins to drift due to the formation of dust. This phenomenon induces strong modifications on the voltage amplitude at the active electrode (see Fig. 4). In the mean time, every element of the matching circuit is kept constant. The plasma bulk impedance is determined according to

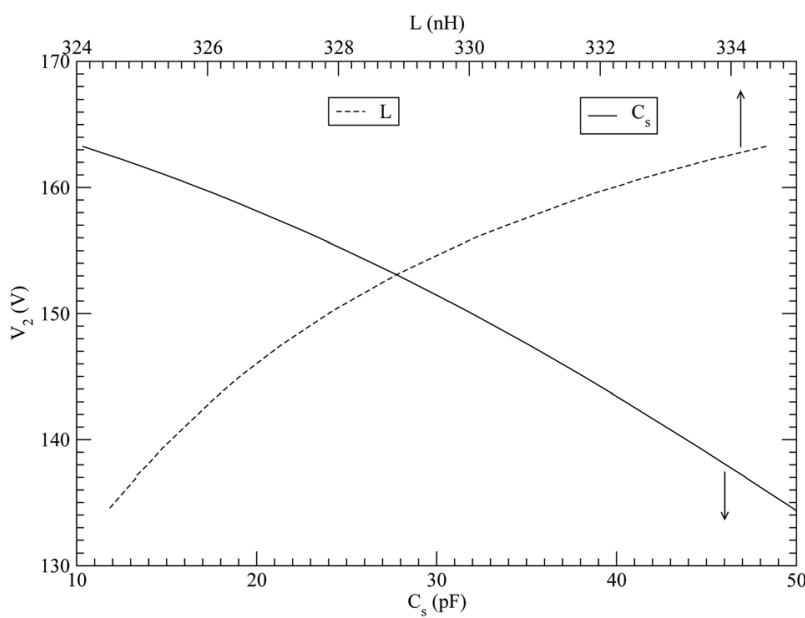

FIG. 8. Relationship between $L, C_{s}$, and $\widehat{V_{2}}$ for an optimized impedance matching.
TABLE I. Parameters of the electrical circuit without dust in the plasma. $P_{g}$ is the power provided by the generator.

\begin{tabular}{lcc}
\hline \hline Name & Value & Unit \\
\hline$C_{1}$ & 1640 & $\mathrm{pF}$ \\
$C_{2}$ & 538 & $\mathrm{pF}$ \\
$C_{s}$ & 28 & $\mathrm{pF}$ \\
$r$ & 1.02 & $\Omega$ \\
$R$ & 25 & $\Omega$ \\
$Z_{g}$ & 50 & $\Omega$ \\
$L$ & 329 & $\mathrm{nH}$ \\
$P_{g}$ & 30 & $\mathrm{~W}$ \\
$P_{l}$ & 29.9 & $\mathrm{~W}$ \\
$P_{p}$ & 2 & $\mathrm{~W}$ \\
\hline \hline
\end{tabular}

the measurement of the mean electron density (see Figs. 3 and 7). Consequently, the sheath capacitance evolution can be determined from Eq. (15), where it is the only remaining unknown. The result is presented in Figure 9 where it is possible to identify four different phases on the evolution of the sheath capacitance during the dust growth. These phases, which are presented in Table II, are correlated with those of the dust formation that are usually identified on the evolution of the electron density. ${ }^{17,30,31}$

Obviously, the evolution of the discharge impedance modifies the coupled power to the load and to the plasma. This is what is presented in Figure 10 where we can see that the power coupled into the plasma increases by a factor of five $(2 \mathrm{~W} \rightarrow 10 \mathrm{~W})$ during the dust formation while the power coupled to the load decreases slightly during the dust growth $(29.9 \mathrm{~W} \rightarrow 27 \mathrm{~W})$ due to the drift of the load impedance. Actually, we found that the plasma coupled power is more or less inversely proportional to the electron density in the reactor, which is in accordance with theory. ${ }^{18}$ Moreover, the amplitude of the radio frequency voltage drop across the plasma bulk is enhanced in the same way as the plasma coupled power during the dust growth (see Fig. 11) leading to a strong increase of the electric field in the plasma. These phenomena trigger the increase of the plasma light emission (Fig. 5), of the ionisation rate and of the electron temperature (Fig. 6) usually observed during a dust growth in a capacitively

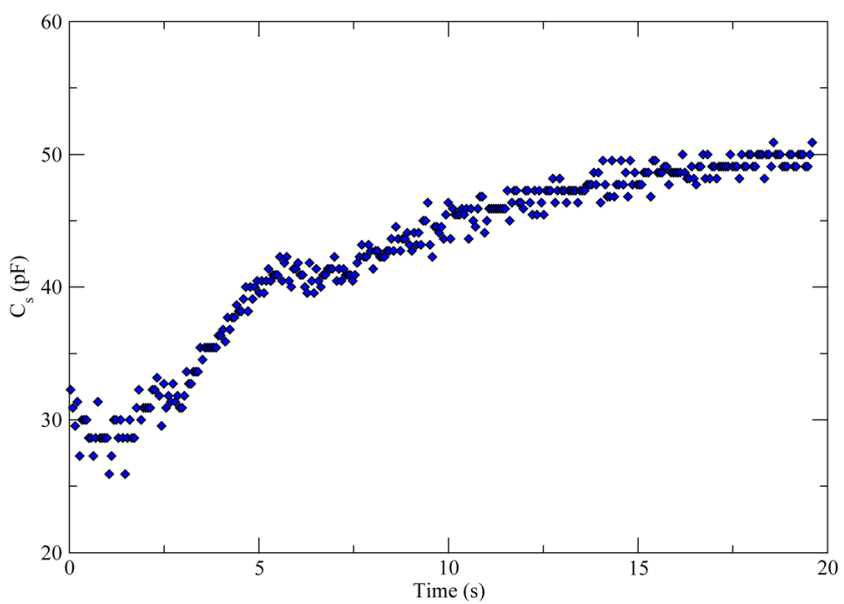

FIG. 9. (Color online) Time evolution of the electrode plasma sheath capacitance. 
TABLE II. Identification of the different dust growth phases.

\begin{tabular}{lcc}
\hline \hline Beginning (s) & End (s) & Dust growth phase \\
\hline 0 & 2 & Methane conversion \\
2 & 3 & Nucleation \\
3 & 6 & Agglomeration \\
6 & 20 & Surface deposition growth \\
\hline
\end{tabular}

coupled radio frequency discharge and reported in many works. $^{17,22,32-36}$

Finally the phase shift between the current and the voltage at the active electrode was computed according to the evolution of the discharge impedance (see Fig. 12). In our conditions, the phase shift evolution is monotonous during the dust formation. This is not what Schauer et al. obtained in their experiments. This discrepancy could be due to the dust particle size and/or concentration which might have been higher in Schauer's experiment than in ours leading to a bigger electron density decrease during the dust formation due to electron attachment on the dust particles. Nevertheless, a comparison can be carried out if we roughly extrapolate our measurements. In fact, we can notice that the plasma bulk resistance is inversely proportional to the mean electron density as follows:

$$
R \approx \frac{2.4 \times 10^{17}}{\overline{n_{e}}}+4.05
$$

and during the last phase of our dust growth $\left(\overline{n_{e}}<3 \times 10^{15} \mathrm{~m}^{-3}\right.$ or $\left.6<t<20 \mathrm{~s}\right)$, the sheath capacitance is proportional to the plasma bulk resistance as follows:

$$
C_{s} \approx 4.4 \times 10^{-13}+6.5 \times 10^{-12},
$$

if we consider this relationship to remain valid for smaller electron densities, the plasma bulk resistance and the plasma sheath capacitance can be extrapolated for smaller electron densities. Then, the phase shift between the current and the voltage at the active electrode can be extrapolated taking into account the stray capacitance $C_{2}$. This extrapolated phase shift is presented in Figure 13 which is in a very good accordance
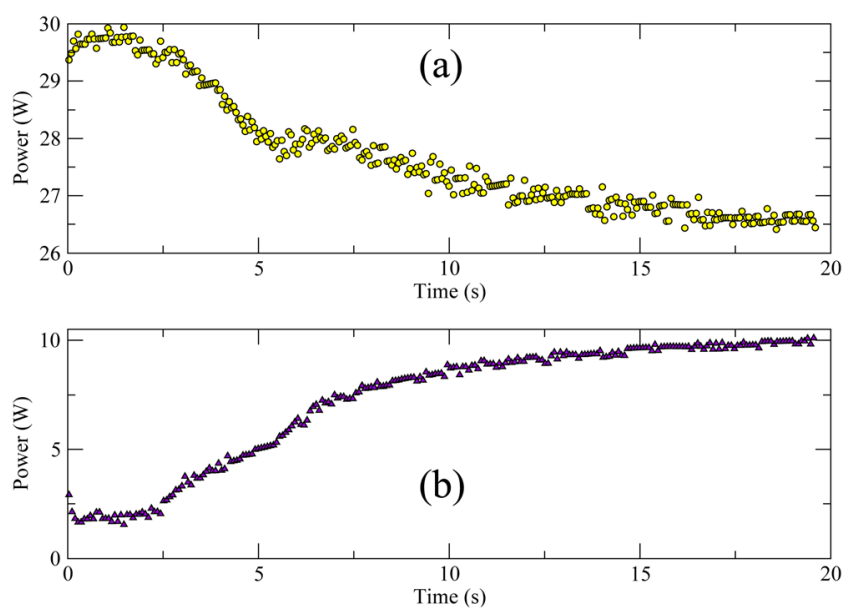

FIG. 10. (Color online) Load (a) and plasma (b) coupled powers evolution.

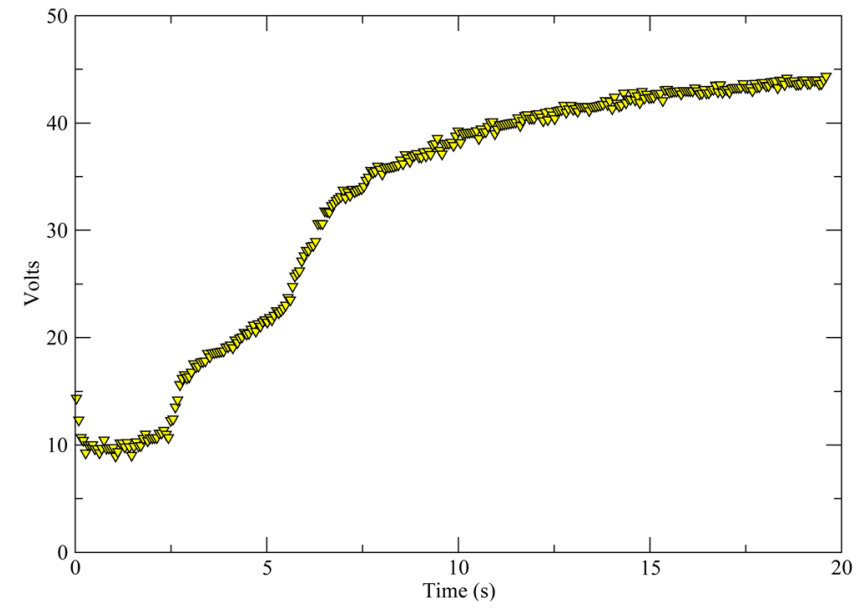

FIG. 11. (Color online) Plasma bulk voltage drop evolution during the dust growth.

with the work of Schauer et al. in the sense that the phase shift evolution with respect to the electron density might be not monotonous during the dust growth if the electron density is small enough (or the dust concentration and/or size are too much important). If we still consider our extrapolation for $R$ and $C_{s}$, the relationship between the electron density and the power coupled to the plasma can be calculated for electron density smaller than $3 \times 10^{15} \mathrm{~m}^{-3}$. As shown in Figure 14, the power coupled to the plasma could reach $50 \%$ of the power delivered by the generator in our experimental conditions, this value being related to an electron density of about $10^{15} \mathrm{~m}^{-3}$. In order to study the effect of the stray capacitance on the power coupled to the plasma, this power is also calculated varying the stray capacitance value into the range $\pm 5 \%$ and calculating which value for $L$ should be set to achieve the impedance matching without dust. The range of variation of the stray capacitance is chosen in order to ensure that our extrapolations are still acceptable. In practice, the stray capacitance value is usually found between $100 \mathrm{pF}$ and several $\mathrm{nF}$. For such values, we have no guarantee that our hypothesis remains acceptable. Yet, it appears in Figure 14 that the effect of the stray capacitance on the power coupled into the plasma is not negligible. Consequently, the stray capacitance should

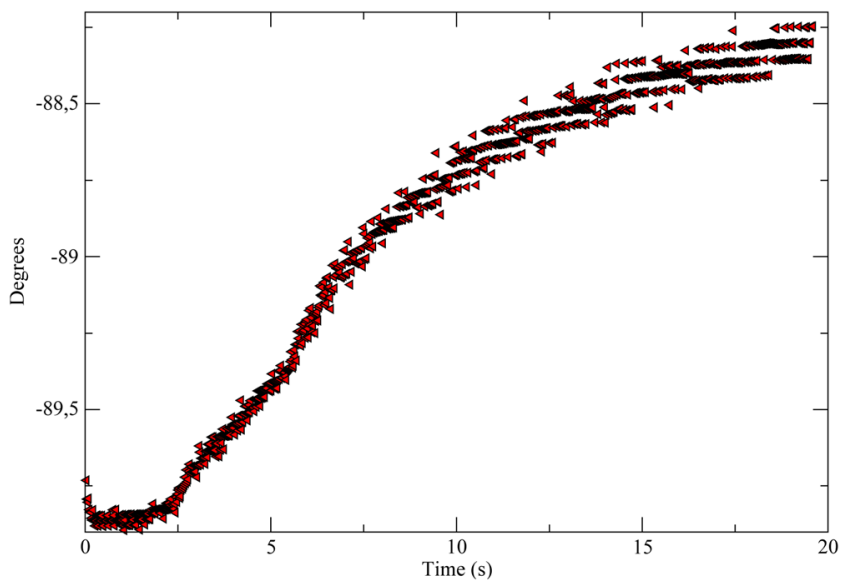

FIG. 12. (Color online) Curent/voltage phase shift at the active electrode versus time. 


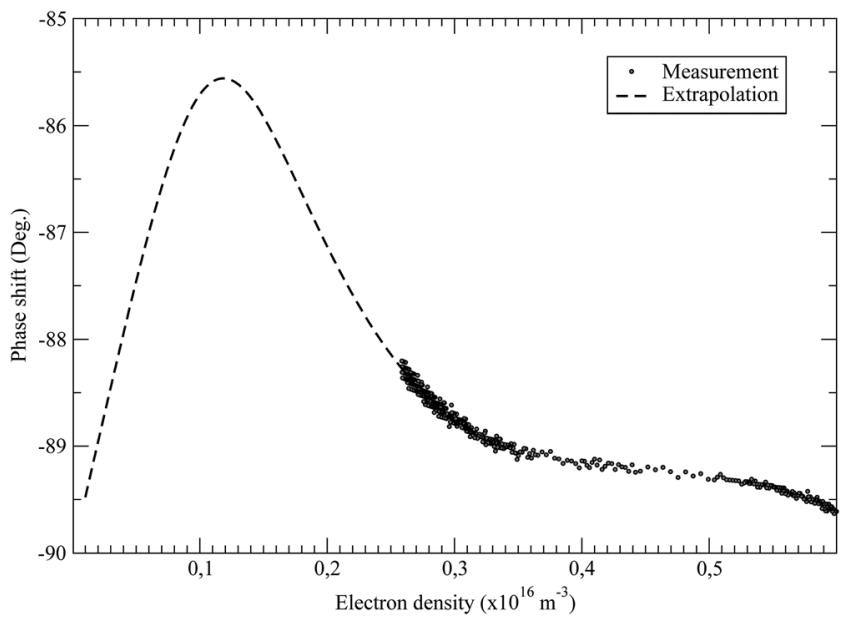

FIG. 13. Active electrode current/voltage phase shift extrapolation versus the electron density.

influence the ionisation rate and the electron temperature evolution that is to say the dust growth kinetic. Finally, this parasitic capacitance might explain the usual discrepancies observed comparing similar experiments carried out on different reactors. This result suggests the necessity to consider the whole electrical circuit if one want to reproduce an experiment on another setup or if one want to model (and then compare with reality) the electrical evolution of the discharge during a dust growth.

\section{DUST PARTICLE SIZE AND SHEATH CAPACITANCE CORRELATION}

As the electron density decreases in the plasma during the dust formation, the plasma electrode sheath width should increase according to dust-free discharge classical theories. ${ }^{18,37-39}$ Actually, we observe even with the naked eye a decrease of the plasma electrode sheath width (which implies an increase of the electrodes sheath capacitance). This experimental observation is in accordance with the evolution of the sheath capacitance determined in Sec. V (see Fig. 9). Thus, it appears that classical (dust-free) models have to take into

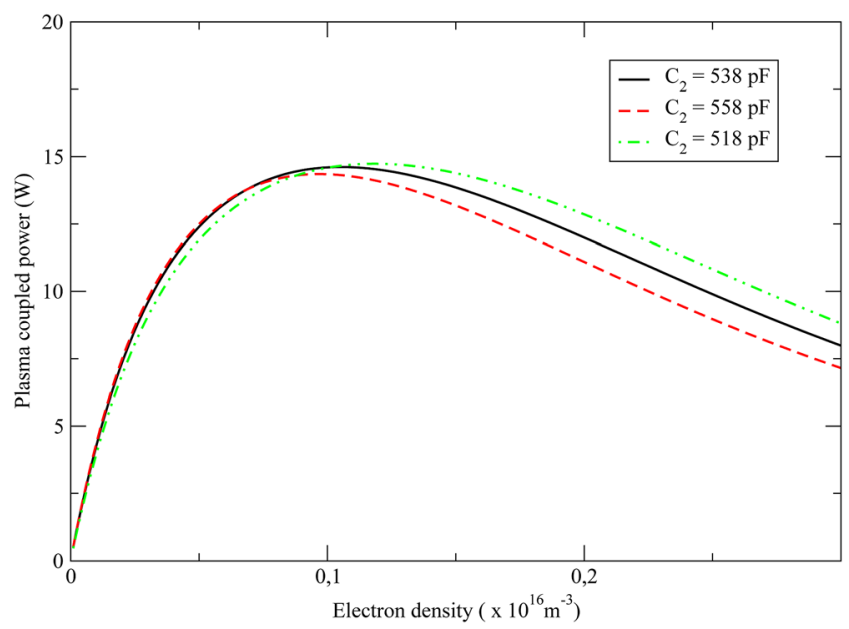

FIG. 14. (Color online) Plasma coupled power extrapolation versus the electron density for different stray capacitances. The power delivered by the generator is $30 \mathrm{~W}$. account the effect of the dust particles on the electrical parameters of the discharge. During our measurements analysis, we noticed that the increase of the sheath capacitance is proportional to the dust particles size. In fact, there is a perfect match between the increase of the sheath capacitance $\left(\Delta C_{s}\right)$ and the ratio between the variation of the self bias $\left(\Delta V_{D C}\right)$ and the variation of the electron density $\left(\Delta n_{e}\right)$. In a previous work, ${ }^{15}$ we have demonstrated that this last ratio is proportional to the dust particle size during the agglomeration and the surface deposition growth phases. The comparison is shown in Figure 15.

After studying different hypothesis in order to explain this phenomenon, we interpret this experimental finding as the result of the interaction between the electric fields of the electrode sheath and of the dust particle sheath. In fact, the dust particles being charged proportionally to their size $\phi_{d}$ (orbital motion limited theory ${ }^{40,41}$ ), those located in the vicinity of the sheath exert an electrostatic force on the sheath. Moreover, it is reasonable to consider the dust particle concentration to remain constant in the vicinity of the electrode/ plasma sheath during the process so the force exerted by the dust particles on the discharge sheath should be proportional to the mean size of the particles located close from the sheath. If we assume that the sheath width variation $\Delta s_{m}$ is proportional to the size of the dust particles, the electrode sheath capacitance (which corresponds mainly to the active electrode sheath capacitance in a strongly asymmetric discharge ${ }^{18}$ ) during the dust growth is given by

$$
\begin{gathered}
C_{s}=\frac{C_{s_{0}}}{1-\frac{\Delta s_{m}}{s_{m_{0}}}} \\
=\frac{C_{s_{0}}}{1-\frac{\alpha \phi_{d}}{s_{m_{0}}}}
\end{gathered}
$$

with $C_{s_{0}}$ and $s_{m_{0}}$ the initial electrode sheath capacitance and the initial sheath thickness at the ignition of the discharge and $\alpha$ is a positive constant. Finally, if the relative variation of the sheath thickness is small such as it is the case for our experiment, a first order Taylor series approximation of

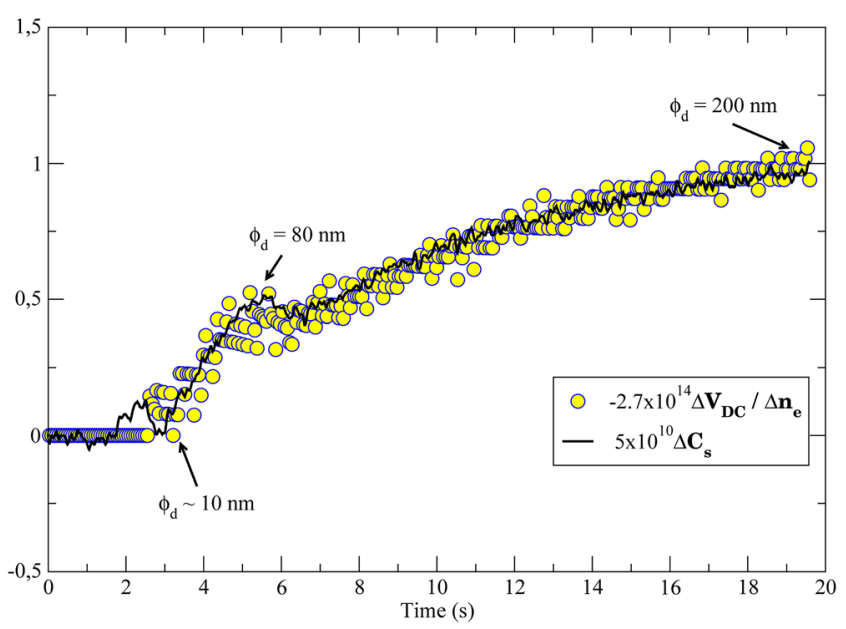

FIG. 15. (Color online) Comparison between $\Delta C_{s}$ and $\frac{\Delta V_{D C}}{n_{e}}\left(\phi_{d}\right.$ is the diameter of the dusts according to Ref. 15). 
Eq. (20) exhibit the observed linear relationship between the electrode sheath capacitance and the size of the dust particles

$$
C_{s} \approx C_{s_{0}}\left(1+\frac{\alpha \cdot \phi_{d}}{s_{m_{0}}}\right) .
$$

\section{CONCLUSION}

This work which was initially performed in order to study a new method to measure the power coupled to the plasma has brought a lot more. We have demonstrated the ability to measure the evolution of the plasma coupled power and of the discharge impedance without measuring the phase shift between the current and the voltage at the active electrode. This method allowed us to follow the evolution of the plasma impedance during the dust formation. We noticed that a strong increase of the power coupled into the plasma occurs due to the dust particles formation. This phenomenon is responsible for the increase of the light emission and of the electron temperature of the plasma.

Moreover, we pointed out the effect of the stray capacitance on the power coupled into the plasma. This capacitance should play a role on the dust particle growth in the reactor and could be responsible for discrepancies between similar experiments.

Finally, we underlined that theoretical models developed for dust-free discharges should be completed in order to take into account the presence of the dust particles in the discharge and we proposed a way to correlate the evolution of the sheath capacitance and the size of the dust particles in dusty plasma. This result should be used by people who want to model dusty plasma sheath.

\section{ACKNOWLEDGMENTS}

This work is supported by ANR for NANOCARA project under No. ANR-08-NANO-044-04 and the French "Conseil Regional, Region Centre" under the Contract No. 2007700026538.

${ }^{1}$ M. Hirt, D. Block, and A. Piel, Phys. Plasmas 11(12), 5690 (2004).

${ }^{2}$ A. V. Ivlev, U. Konopka, G. Morfill, and G. Joyce, Phys. Rev. E 68, 026405 (2003).

${ }^{3}$ A. P. Nefedov, G. E. Morfill, V. E. Fortov, H. M. Thomas, H. Rothermel, T. Hagl, A. V. Ivlev, M. Zuzic, B. A. Klumov, A. M. Lipaev, V. I. Molotkov, O. F. Petrov, Y. P. Gidzenko, S. K. Krikalev, W. Shepherd, A. I. Ivanov, M. Roth, H. Binnenbruck, J. A. Goree, and Y. P. Semenov, New J. Phys. 5(1), 33 (2003).

${ }^{4}$ Y. Nakamura and H. Bailung, Rev. Sci. Instrum. 70(5), 2345 (1999)

${ }^{5}$ J. Winter, Plasma Phys. Controlled Fusion 46 (12B), B583 (2004).

${ }^{6} \mathrm{~K}$. D. Bleecker, "Modeling of the formation of nanoparticles in dusty plasmas," Ph.D. dissertation (Universiteit Antwerpen, 2006).

${ }^{7}$ G. S. Selwyn, J. E. Heidenreich, and K. L. Haller, J. Vac. Sci. Technol. A 9(5), 2817 (1991).

${ }^{8}$ R. M. Roth, K. G. Spears, G. D. Stein, and G. Wong, Appl. Phys. Lett. 46(3), 253 (1985).

${ }^{9}$ R. L. Merlino, "Dusty plasmas and applications in space and industry," Transworld Research Network 37/661 (2), Fort P.O., Trivandrum 695023 , Kerala, India, 2006.
${ }^{10}$ International Technology Roadmap for Semiconductors, Technical Report, 2009.

${ }^{11}$ P. Roca i Cabarrocas, N. Chaâbane, A. V. Kharchenko, and S. Tchakarov, Plasma Phys. Controlled Fusion 46(12B), B235 (2004).

${ }^{12}$ S. Veprek, S. Reiprich, and L. Shizhi, Appl. Phys. Lett. 66(20), 2640 (1995).

${ }^{13}$ E. Stoffels, W. W. Stoffels, G. Ceccone, R. Hasnaoui, H. Keune, G. Wahl, and F. Rossi, J. Appl. Phys. 86(6), 3442 (1999).

${ }^{14}$ D. Zhou, T. G. McCauley, L. C. Qin, A. R. Krauss, and D. M. Gruen, J. Appl. Phys. 83(1), 540 (1998).

${ }^{15}$ G. Wattieaux, A. Mezeghrane, and L. Boufendi, Phys. Plasmas 18(9), 093701 (2011).

${ }^{16}$ G. Wattieaux, P. Lecerf, L. Meyer, L. Boufendi, Y. Leconte, O. Sublemontier, N. Herlin, A. Asimakopoulou, A. Tsakis, M. Daskalos, A. G. Konstandopoulos, and F. X. Ouf, J. Phys.: Conf. Ser. 304(1), 012021 (2011).

${ }^{17}$ L. Boufendi, A. Bouchoule, and T. Hbid, J. Vac. Sci. Technol. A 14(2), 572 (1996)

${ }^{18}$ M. Lieberman and A. Lichtenberg, Principle of Plasma Discharges and Materials Processing (Wiley, New York, 2005), p. 456.

${ }^{19}$ T. Nelis, M. Aeberhard, L. Rohr, J. Michler, P. Belenguer, P. Guillot, and L. Thérèse, Anal. Bioanal. Chem. 389, 763 (2007).

${ }^{20}$ F. Canpont, Ph.D. dissertation (Université Claude Bernard, Lyon, France, 1993).

${ }^{21}$ K. A. Marshall, T. J. Casper, K. R. Brushwyler, and J. C. Mitchell, J. Anal. At. Spectrom. 18, 637 (2003).

${ }^{22}$ A. Bouchoule and L. Boufendi, Plasma Sources Sci. Technol. 2, 204 (1993).

${ }^{23}$ S. C. Brown and J. C. Ingraham, "Plasma diagnostics-Technical Report No. 454," Technical Report, Massachusetts Institute of Technology Research Laboratory of Electronics, 1966.

${ }^{24}$ R. Papoular and J. Balazard, Applications Des Ondes Hyperfréquences Et Infrarouges á L'étude Des Plasmas (Dunod, Paris, 1965).

${ }^{25}$ Yu. Ralchenko, A. E. Kramida, J. Reader, and NIST ASD Team (2008), NIST Atomic Spectra Database (version 3.1.5), National Institute of Standards and Technology, Gaithersburg, MD, see http://physics.nist.gov/ asd3 (last accessed August 18, 2010).

${ }^{26}$ U. Fantz, Plasma Sources Sci. Technol. 15(4), S137 (2006).

${ }^{27}$ F. J. Gordillo-Vasquez, M. Camero, and C. Gomez-Aleixandre, Plasma Sources Sci. Technol. 15(1), 42 (2006).

${ }^{28}$ V. Godyak, R. Piejak, and B. Alexandrovich, IEEE Trans. Plasma Sci. 4, 660 (1991).

${ }^{29}$ J.-C. Schauer, S. Hong, and J. Winter, Plasma Sources Sci. Technol. 13(4), 636 (2004)

${ }^{30}$ L. Boufendi, A. Bouchoule, and T. Hbid, "Electrical characterization and modeling of a dust forming plasma in a radio frequency discharge," in Dusty Plasmas -'95 Workshop on Generation, Transport, and Removal of Particles in Plasmas (AIP, New York, 1996), Vol. 14, pp. 572-576.

${ }^{31}$ A. A. Fridman, L. Boufendi, T. Hbid, B. V. Potapkin, and A. Bouchoule, J. Appl. Phys. 79(3), 1303 (1996).

${ }^{32}$ I. Denysenko, J. Berndt, E. Kovacevic, I. Stefanovic, V. Selenin, and J. Winter, Phys. Plasmas 13, 073507 (2006).

${ }^{33}$ M. Mikikian, L. Couëdel, M. Cavarroc, Y. Tessier, and L. Boufendi, New J. Phys. 9(8), 268 (2007).

${ }^{34}$ I. I. Stefanovic, E. Kovacevic, J. Berndt, and J. Winter, J. Phys.: Conf. Ser. 71, 012015 (2007).

${ }^{35} \mathrm{C}$. Chen, "Electron temperature enhancement effects on plasma irregularities associated with charged dust in the earth's mesosphere," Ph.D. dissertation (Virginia Polytechnic Institute, Blacksburg, Virginia, 2007).

${ }^{36}$ P. Agarwal, J. Berndt, E. Kovacevic, L. Boufendi, and S. L. Girshick, "Rf dusty plasmas in which nanoparticles nucleate and grow: Comparison of numerical simulations and experiments," in 30th International Conference on Phenomena in Ionized Gases (ICPIG), 2011.

${ }^{37}$ V. A. Godyak and N. Sternberg, Phys. Rev. A 42, 2299 (1990).

${ }^{38}$ F. F. Chen, Plasma Sources Sci. Technol. 15(4), 773 (2006).

${ }^{39}$ T. E. Sheridan and J. Goree, Phys. Fluids B 3(10), 2796 (1991).

${ }^{40}$ I. Langmuir, Collected Works of Irving Langmuir (General Electric, Pergamon, New York, 1961).

${ }^{41}$ I. Langmuir and H. M. M. Smith, Phys. Rev. 28, 727 (1926). 